\title{
Regio- and Stereoselective Copper(II)-Catalyzed Hydrosilylation of Activated Allenes in Water: Access to Vinylsilanes
}

Srinath Pashikanti, Joseph A. Calderone, Matthew K. Nguyen, Christopher D. Sibley, and

\author{
Webster L. Santos* \\ Department of Chemistry, Virginia Tech, Blacksburg, Virginia 24061, United States \\ E-mail: santosw@vt.edu
}

\section{Table of Contents}

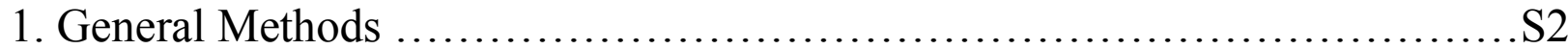

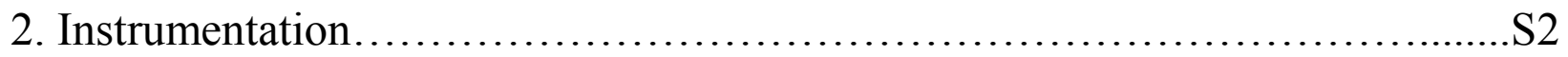

3. General procedure for the synthesis of allenoate substrates...................S3

4. General procedure and characterization of silylation of

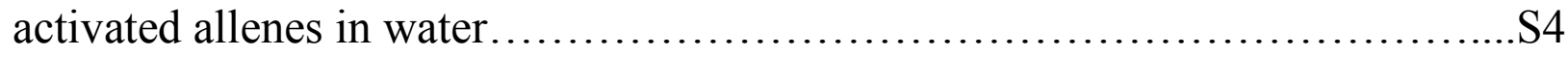

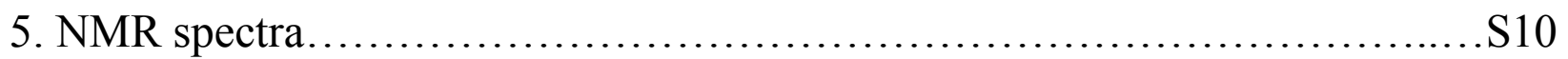

6. References....................................................... 25 


\section{General Methods}

All reactions were carried out open to air, at room temperature, and performed in duplicate. Nuclease-free water was purchased from Qualgen and used as received for preparing aqueous solutions. Dimethylphenylsilylpinacol borane (pinBSiPhMe $)_{2}$ was purchased from either Sigma Aldrich or Frontier Scientific and used as received. Copper sulfate pentahydrate, 4picoline, and other commercially available bases, reagents, and substrates were purchased and used as received. $\mathrm{CuSO}_{4}$ stock solution was prepared by dissolving $\mathrm{CuSO}_{4}$ in nuclease free water. TLC analyses were performed using EMD silica gel $60 \mathrm{~F}_{254}$ plates or Silicycle SiliaPlate $200 \mu \mathrm{M} \mathrm{F}_{254}$ and spots were visualized with permanganate stain.

\section{Instrumentation}

${ }^{1} \mathrm{H}$ NMR spectra were recorded on either a Bruker AvanceII $500 \mathrm{MHz}$, Agilent 400-MR $400 \mathrm{MHz}$, or a Varian Inova $400 \mathrm{MHz}$ spectrometer. Chemical shifts are reported in ppm with the solvent resonance as the internal standard $\left(\mathrm{CDCl}_{3}: 7.26 \mathrm{ppm}\right)$. Data are reported as follows: chemical shift, multiplicity $(\mathrm{s}=$ singlet, $\mathrm{d}=$ doublet, $\mathrm{t}=$ triplet, $\mathrm{q}=$ quarter, $\mathrm{m}=$ multiplet $)$, coupling constants $(\mathrm{Hz})$, and integration. ${ }^{13} \mathrm{C}$ NMR spectra were recorded on a Bruker AvanceII $500 \mathrm{MHz}$, Agilent 400-MR or a Varian Inova $400 \mathrm{MHz}$ spectrometer. Chemical shifts are reported in ppm with the solvent resonance as the internal standard $\left(\mathrm{CDCl}_{3}: 77.16 \mathrm{ppm}\right)$. Normal and high resolution mass spectra (HRMS) were performed on an Agilent LC-ESI-TOF. Gas chromatography (GC) analyses were performed on a Hewlett Packard 6890 Series GC system coupled to a HP 5973 Mass Selective Detector or Agilent 7890 Series GC system coupled to a HP 5975 Mass Selective Detector. The column was an Agilent DB-5MS with a length of $30 \mathrm{~m}$, I.D. of $250 \mu \mathrm{m}$ and film thickness of $0.25 \mu \mathrm{m}$. 


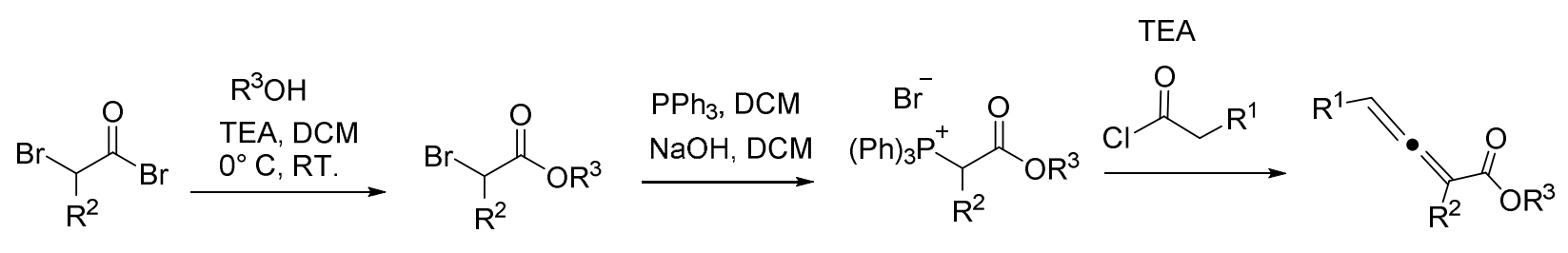

2b-h,2j-n

Allenoate substrates were prepared following a reported procedure by Kwon and from our group, as described above ${ }^{1}$. Spectral characterization of $\mathbf{2} \mathbf{b}-\mathbf{h}, \mathbf{2} \mathbf{j}-\mathbf{n}$ is in agreement with the reported allenoate substrates as shown in the table S1.

Table S1. References for allenoate substrates

\begin{tabular}{|l|l|l|l|l|}
\hline Substrate & $\mathbf{R}^{1}$ & \multicolumn{1}{|c|}{$\mathbf{R}^{2}$} & \multicolumn{1}{|c|}{$\mathbf{R}^{3}$} & \multicolumn{1}{|c|}{ Reference } \\
\hline $2 \mathrm{a}$ & $\mathrm{H}$ & $\mathrm{H}$ & $\mathrm{Et}$ & Available from Sigma Aldrich \\
\hline $2 \mathrm{~b}$ & $\mathrm{H}$ & $\mathrm{H}$ & $\mathrm{Bn}$ & J. Am. Chem. Soc. 2002, 124, 13646 \\
\hline $2 \mathrm{c}$ & $\mathrm{H}$ & $\mathrm{H}$ & $\mathrm{Ph}$ & J. Am. Chem. Soc. 2009, 131, 6105 \\
\hline $2 \mathrm{~h}$ & $\mathrm{H}$ & $\mathrm{Me}$ & $\mathrm{Et}$ & J. Am. Chem. Soc. 2001, 123, 12466 \\
\hline $2 \mathrm{i}$ & $\mathrm{H}$ & $\mathrm{CH}_{2} \mathrm{C}(\mathrm{O}) \mathrm{OEt}$ & $\mathrm{Et}$ & Available from Sigma Aldrich \\
\hline $2 \mathrm{j}$ & $\mathrm{Me}$ & $\mathrm{H}$ & $\mathrm{Bn}$ & J. Am. Chem. Soc. 2002, 124, 13646 \\
\hline $2 \mathrm{k}$ & $\mathrm{Me}$ & $\mathrm{H}$ & $-O-\mathrm{NO}_{2} \mathrm{Bn}$ & J. Chem. Commun. 2011, 47, 424-426 \\
\hline $2 \mathrm{l}$ & $\mathrm{Ph}$ & $\mathrm{H}$ & $\mathrm{Et}$ & J. Am. Chem. Soc. 2007, 129, 1494 \\
\hline $2 \mathrm{~m}$ & $\mathrm{Ph}$ & $\mathrm{H}$ & ${ }^{i} \mathrm{Pr}$ & J.Chem. Commun. 2011, 47, 424-426 \\
\hline $2 \mathrm{n}$ & $\mathrm{Ph}$ & $\mathrm{H}$ & $\mathrm{Bn}$ & Eur. J. Org. Chem. 2013, 3901-3909 \\
\hline
\end{tabular}




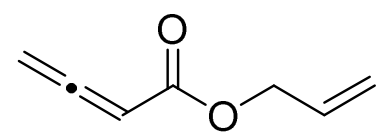

Allyl buta-2,3-dienoate (2d) The title compound was isolated in $16 \%$ yield $(50 \mathrm{mg})$ as a colorless oil. TLC $\mathrm{R}_{\mathrm{f}}=0.45$ in $98: 2$ / hexanes:EtOAc.

${ }^{1} \mathrm{H} \mathrm{NMR}\left(400 \mathrm{MHz}, \mathrm{CDCl}_{3}\right) \delta 5.91-5.84(\mathrm{~m}, 1 \mathrm{H}), 5.64(\mathrm{t}, J=6.4 \mathrm{~Hz}, 1 \mathrm{H}), 5.35-5.28(\mathrm{~m}, 1 \mathrm{H})$, $5.28(\mathrm{~s}, 0.5 \mathrm{H}), 5.25-5.20(\mathrm{~m}, 2.5 \mathrm{H}), 4.65-4.61(\mathrm{~m}, 2 \mathrm{H})$.

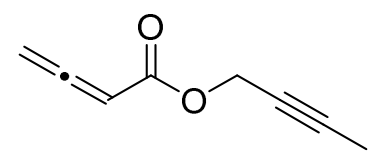

But-2-yn-1-yl buta-2,3-dienoate (2g)The title compound was isolated in $37 \%$ yield $(95 \mathrm{mg})$ as a colorless oil. TLC $\mathrm{R}_{\mathrm{f}}=0.4$ in $95: 5 /$ hexanes:EtOAc. ${ }^{1} \mathrm{H}$ NMR $\left(500 \mathrm{MHz}, \mathrm{CDCl}_{3}\right) \delta 5.69(\mathrm{t}, J=6.5 \mathrm{~Hz}, 1 \mathrm{H}), 5.29(\mathrm{~d}, J=6.5 \mathrm{~Hz}$, 2H), $4.74(\mathrm{~s}, 2 \mathrm{H}), 1.88(\mathrm{~s}, 3 \mathrm{H})$.

\section{General procedure and characterization of silylation of activated allenes in water}

In a 1 dram vial, $\alpha, \beta$ - unsaturated allenoate (1.0 equivalent), 4-picoline ( 0.05 equivalent). $\mathrm{Me}_{2} \mathrm{PhSiBPin}$ (1.1 or 1.5 equivalent), and $1 \mathrm{~mL}$ of $1.3 \mathrm{mgmL}^{-1} \mathrm{CuSO}_{4}$ stock solution $(0.01$ equivalent) were vigorously stirred at room temperature. The reaction was quenched with $1 \mathrm{~mL}$ of hexanes as followed by TLC. The aqueous layer was extracted $3 \mathrm{x}$ with hexanes. The combined hexanes layer was back extracted $5 \mathrm{x}$ with water and then dried over sodium sulfate. Silica gel chromatography was used to purify the product.

$\mathrm{Me}_{2} \mathrm{PhSi} \quad \mathrm{O}$ Ethyl 3-(dimethyl(phenyl)silyl)but-3-enoate (3a) The title compound was isolated in a $76 \%$ yield $(99 \mathrm{mg})$ as a clear oil $\left(\mathrm{bp}=285^{\circ} \mathrm{C}\right) . \quad \mathrm{TLC} \mathrm{R}_{\mathrm{f}}=0.52$ in 90:10 / hexanes:EtOAc. ${ }^{1} \mathrm{H}$ NMR $\left(400 \mathrm{MHz}, \mathrm{CDCl}_{3}\right) \delta 7.57-7.46(\mathrm{~m}, 2 \mathrm{H}), 7.39-7.31(\mathrm{~m}$, 
$3 \mathrm{H}), 5.87-5.82(\mathrm{~m}, 1 \mathrm{H}), 5.59-5.56(\mathrm{~m}, 1 \mathrm{H}), 4.00(\mathrm{q}, J=12,8 \mathrm{~Hz}, 2 \mathrm{H}), 3.09(\mathrm{~s}, 2 \mathrm{H}), 1.17(\mathrm{t}, J$ $=7.2 \mathrm{~Hz}, 3 \mathrm{H}), 0.39(\mathrm{~s}, 6 \mathrm{H})$. HRMS (ESI+): Calcd for $\mathrm{C}_{14} \mathrm{H}_{21} \mathrm{O}_{2} \mathrm{Si}[\mathrm{M}+\mathrm{H}]^{+}:$249.1305, Found: 249.1302. ${ }^{1} \mathrm{H}$ NMR is consistent with the literature. ${ }^{2}$

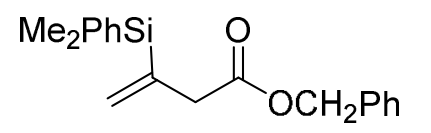

Benzyl 3-(dimethyl(phenyl)silyl)but-3-enoate (3b) The title compound was isolated in a $96 \%$ yield $(77 \mathrm{mg})$ as a clear oil ( $\mathrm{bp}=361$

$\left.{ }^{\circ} \mathrm{C}\right)$. TLC $\mathrm{R}_{\mathrm{f}}=0.61$ in 90:10 / hexanes:EtOAc. ${ }^{1} \mathrm{H}$ NMR (400 MHz, $\left.\mathrm{CDCl}_{3}\right) \delta 7.52-7.46(\mathrm{~m}$, 2H), $7.39-7.27(\mathrm{~m}, 7 \mathrm{H}), 5.88-5.82(\mathrm{~m}, 1 \mathrm{H}), 5.61-5.56(\mathrm{~m}, 1 \mathrm{H}), 4.98(\mathrm{~s}, 2 \mathrm{H}), 3.15(\mathrm{t}, J=1.6$ $\mathrm{Hz}, 2 \mathrm{H}), 0.37$ (s, 6H); ${ }^{13} \mathrm{C}$ NMR $\left(101 \mathrm{MHz}, \mathrm{CDCl}_{3}\right) \delta 171.8,142.9,137.4,136,134.1,130.7$, 129.3, 128.6, 128.4, 128.3, 127.9, 66.5, 41.8, -2.9. HRMS (ESI+): Calcd for $\mathrm{C}_{19} \mathrm{H}_{22} \mathrm{O}_{2} \mathrm{Si}$ $[\mathrm{M}+\mathrm{Na}]^{+}: 333.1281$, Found: 333.1306.

$\mathrm{Me}_{2} \mathrm{PhSi} \quad \mathrm{O}$ Phenyl 3-(dimethyl(phenyl)silyl)but-3-enoate (3c) The title OPh compound was isolated in $83 \%$ yield $(64 \mathrm{mg})$ as a clear oil. TLC $\mathrm{R}_{\mathrm{f}}=$ 0.423 in 90:10 / hexanes:EtOAc. ${ }^{1} \mathrm{H}$ NMR (400 MHz, $\left.\mathrm{CDCl}_{3}\right) \delta 7.57-7.54(\mathrm{~m}, 2 \mathrm{H}), 7.42-7.30$ (m, 5H), $7.23-7.16(\mathrm{~m}, 2 \mathrm{H}), 6.96-6.91(\mathrm{~m}, 2 \mathrm{H}), 6.01-5.95(\mathrm{~m}, 1 \mathrm{H}), 5.71-5.66(\mathrm{~m}, 1 \mathrm{H}), 3.35$ $-3.31(\mathrm{~s}, 2 \mathrm{H}), 0.47-0.44(\mathrm{~s}, 6 \mathrm{H}) ;{ }^{13} \mathrm{C} \mathrm{NMR}\left(101 \mathrm{MHz}, \mathrm{CDCl}_{3}\right) \delta 170.2,150.7,142.4,137.1$, 134, 130.9, 129.3, 129.2, 127.9, 125.7, 121.4, 41.51, -3. HRMS (ESI+): Calcd for $\mathrm{C}_{18} \mathrm{H}_{21} \mathrm{O}_{2} \mathrm{Si}$ $[\mathrm{M}+\mathrm{H}]^{+}:$297.4443, Found: 297.1307.

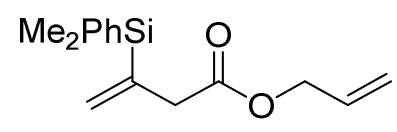

Allyl 3-(dimethyl(phenyl)silyl)but-3-enoate (3d) The title compound was isolated in a $30 \%$ yield $(37 \mathrm{mg})$ as a clear oil $\left(\mathrm{bp}>250{ }^{\circ} \mathrm{C}\right.$ (decomp). TLC $\mathrm{R}_{\mathrm{f}}=0.48$ in 98:2/ hexanes:EtOAc. ${ }^{1} \mathrm{H}$ NMR (500 MHz, $\left.\mathrm{CDCl}_{3}\right) \delta 7.46-7.36(\mathrm{~m}$, 
2H), $7.34-7.21(\mathrm{~m}, 3 \mathrm{H}), 5.80-5.70(\mathrm{~m}, 2 \mathrm{H}), 5.53-5.50(\mathrm{~s}, 1 \mathrm{H}), 5.21-5.09(\mathrm{~m}, 2 \mathrm{H}), 4.39-$ $4.30(\mathrm{~m}, 2 \mathrm{H}), 3.05(\mathrm{~s}, 2 \mathrm{H}), 0.32(\mathrm{~s}, 6 \mathrm{H}) ;{ }^{13} \mathrm{C} \mathrm{NMR}\left(101 \mathrm{MHz}, \mathrm{CDCl}_{3}\right) \delta 171.4,142.7,137.3$, $134.1,134,132.1,130.5,129.2,128.5,127.8,127.7,118.1,83.4,65.2,41.6,25,-3$. GCMS (m/z): Calcd for $\mathrm{C}_{15} \mathrm{H}_{20} \mathrm{O}_{2} \mathrm{Si}[\mathrm{M}]^{+}: 260.1233$, Found: $245.0\left(\mathrm{M}-\mathrm{CH}_{3}\right), 183.0(\mathrm{M}-\mathrm{Ph})$.

$\mathrm{Me}_{2} \mathrm{PhSi} \quad \mathrm{O} \quad$ But-2-yn-1-yl 3-(dimethyl(phenyl)silyl)but-3-enoate (3g) The title<smiles>C=C(C)CC(=O)OCC#CC</smiles>
compound was isolated in a $60 \%$ yield $(120 \mathrm{mg})$ as a clear oil $\left(\mathrm{bp}=310^{\circ} \mathrm{C}\right)$. TLC $\mathrm{R}_{\mathrm{f}}=0.51$ in 98:2/ hexanes:EtOAc. ${ }^{1} \mathrm{H}$ NMR $\left(500 \mathrm{MHz}, \mathrm{CDCl}_{3}\right) \delta 7.55-$ $7.45(\mathrm{~m}, 2 \mathrm{H}), 7.39-7.28(\mathrm{~m}, 3 \mathrm{H}), 5.87-5.78(\mathrm{~m}, 1 \mathrm{H}), 5.60-5.55(\mathrm{~m}, 1 \mathrm{H}), 4.50-4.47(\mathrm{~m}$,

2H), $3.13-3.09(\mathrm{~m}, 2 \mathrm{H}), 1.83(\mathrm{t}, J=8 \mathrm{~Hz}, 2 \mathrm{H}), 0.39(\mathrm{~s}, 6 \mathrm{H}) ;{ }^{13} \mathrm{C} \mathrm{NMR}\left(101 \mathrm{MHz}, \mathrm{CDCl}_{3}\right) \delta$ 171.4, 142.5, 137.2, 134.0, 130.6, 129.1, 127.8, 83.1, 73.1, 61.5, 52.8, 41.4, 3.6, -3.1 GCMS (m/z): Calcd for $\mathrm{C}_{16} \mathrm{H}_{20} \mathrm{O}_{2} \mathrm{Si}[\mathrm{M}]^{+}:$272.1233, Found: $257.1\left(\mathrm{M}-\mathrm{CH}_{3}\right), 195.1(\mathrm{M}-\mathrm{Ph})$.

$\mathrm{Me}_{2} \mathrm{PhSi} \quad \mathrm{O}$ Ethyl 3-(dimethyl(phenyl)silyl)-2-methylbut-3-enoate (3h) The title compound was isolated in a $76 \%$ yield $(46.5 \mathrm{mg})$ as a clear oil $(\mathrm{bp}=281$ $\left.{ }^{\circ} \mathrm{C}\right)$. TLC $\mathrm{R}_{\mathrm{f}}=0.651$ in 90:10/ hexanes:EtOAc. ${ }^{1} \mathrm{H}$ NMR $\left(500 \mathrm{MHz}, \mathrm{CDCl}_{3}\right) \delta 7.56-7.48(\mathrm{~m}$, 2H), $7.39-7.31(\mathrm{~m}, 3 \mathrm{H}), 5.93-5.87(\mathrm{~s}, 1 \mathrm{H}), 5.57(\mathrm{~s}, 1 \mathrm{H}), 4.06-3.89(\mathrm{~m}, 2 \mathrm{H}), 3.30-3.15(\mathrm{~m}$, 1H), $1.21-1.13(\mathrm{~m}, 6 \mathrm{H}), 0.41(\mathrm{~s}, 6 \mathrm{H}) ;{ }^{13} \mathrm{C} \mathrm{NMR}\left(101 \mathrm{MHz}, \mathrm{CDCl}_{3}\right) \delta 174.9,149.4,137.8$ 134.2, 129.2, 128, 127.8, 60.5, 44, 17.9, 1 4.2, -2.5; HRMS (ESI+): Calcd for $\mathrm{C}_{15} \mathrm{H}_{23} \mathrm{O}_{2} \mathrm{Si}$ $[\mathrm{M}+\mathrm{H}]^{+}:$263.1462, Found: 263.1488 . 
$\mathrm{Me}_{2} \mathrm{PhS}$<smiles>C=C(CC(C(=O)OCC)C(=O)OCC)C(=O)O</smiles>

(3i) The title compound was isolated in $81 \%$ yield $(67 \mathrm{mg})$ as a clear oil (bp $\left.=338{ }^{\circ} \mathrm{C}\right)$. TLC $\mathrm{R}_{\mathrm{f}}=0.46$ in 90:10/ hexanes:EtOAc. ${ }^{1} \mathrm{H}$ NMR $(400 \mathrm{MHz}$, $\left.\mathrm{CDCl}_{3}\right) \delta 7.53-7.48(\mathrm{~m}, 2 \mathrm{H}), 7.37-7.31(\mathrm{~m}, 3 \mathrm{H}), 5.90(\mathrm{~s}, 1 \mathrm{H}),, 5.59(\mathrm{~s}, 1 \mathrm{H}), 4.10-3.90(\mathrm{~m}$, 4H), $3.64-3.58(\mathrm{~m}, 1 \mathrm{H}), 2.89-2.81(\mathrm{~m}, 1 \mathrm{H}), 2.35-2.26(\mathrm{~m}, 1 \mathrm{H}), 1.23-1.13(\mathrm{~m}, 6 \mathrm{H}), 0.44-$ $0.41(\mathrm{~d}, 6 \mathrm{H}) ;{ }^{13} \mathrm{C}$ NMR $\left(101 \mathrm{MHz}, \mathrm{CDCl}_{3}\right) \delta 173.2,171.8,146.9,137.3,134.1,129.6,129.4$, 127.9, 60.9, 60.7, 45.5, 37.1, 14.3, 14.1, -2.7; HRMS (ESI+): Calcd for $\mathrm{C}_{18} \mathrm{H}_{26} \mathrm{O}_{4} \mathrm{Si}[\mathrm{M}+\mathrm{Na}]^{+}$: 357.1493, Found: 357.1514.

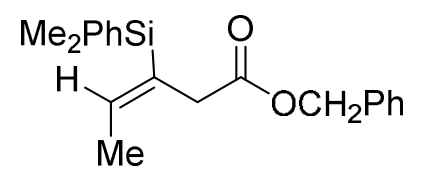

Benzyl (E)-3-(dimethyl(phenyl)silyl)pent-3-enoate (3j) Mixture of regioisomers $(E: Z: 87: 13)$. The title compound was isolated in a $95 \%$

yield $(80 \mathrm{mg})$ as a clear oil. ${ }^{1} \mathrm{H}$ NMR $\left(500 \mathrm{MHz}, \mathrm{CDCl}_{3}\right) \delta$ 7.51- $7.45(\mathrm{~m}, 2 \mathrm{H})$, 7.37-7.26 (m, $8 \mathrm{H}), 6.20-6.11(\mathrm{~m}, 1 \mathrm{H}), 4.97(\mathrm{~s}, 2 \mathrm{H}), 3.18(\mathrm{~s}, 2 \mathrm{H}), 1.72-1.78(\mathrm{~d}, J=6.4 \mathrm{~Hz} .3 \mathrm{H}), 0.34(\mathrm{~s}, 6 \mathrm{H})$; ${ }^{13} \mathrm{C}$ NMR $\left(101 \mathrm{MHz}, \mathrm{CDCl}_{3}\right) \delta 171.8,143.4,140.9,138.2,136.2,134.2,134,132.6,129.1$, $128.6,128.5,128.3,128.2,127.9,127.8,66.4,46.5,34.7,18.5,15.3,-1.3,-2.9$; HRMS (ESI+): Calcd for $\mathrm{C}_{20} \mathrm{H}_{28} \mathrm{O}_{2} \mathrm{NSi}\left[\mathrm{M}+\mathrm{NH}_{4}\right]^{+}: 342.1884$, Found: 342.1914 .

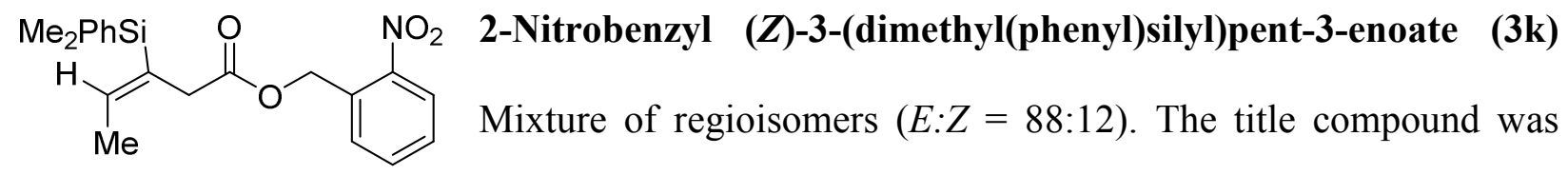
isolated in a 51\% yield $(49 \mathrm{mg})$ as a clear oil. ${ }^{1} \mathrm{H}$ NMR $\delta 8.11-8.06(\mathrm{~m}, 1 \mathrm{H}), 7.63-7.57(\mathrm{~m}$, 1H), $7.52-7.42(\mathrm{~m}, 4 \mathrm{H}), 7.34-7.27(\mathrm{~m}, 3 \mathrm{H}), 6.23-6.16(\mathrm{~m}, 1 \mathrm{H}), 5.44(\mathrm{~s}, 0.2 \mathrm{H}) 5.34(\mathrm{~s}$, 1.8H), $3.23(\mathrm{~s}, 2 \mathrm{H}), 1.78(\mathrm{~d}, J=8 \mathrm{~Hz}, 2.7 \mathrm{H}), 1.68(\mathrm{~d}, J=8 \mathrm{~Hz}, 0.3 \mathrm{H}), 0.41(\mathrm{~s}, 0.6 \mathrm{H}), 0.35$ (s, 5.4H); ${ }^{13} \mathrm{C} \mathrm{NMR}\left(101 \mathrm{MHz}, \mathrm{CDCl}_{3}\right) \delta 171.2,143.7,141.1,138,134.2,134,133.7,132.4,132.3$, 
129.1 128.7, 127.9, 127.8, 125.1, 63, 43.5, 34.5, 29.8, 15.3, -1.3, -2.9; HRMS (ESI+): Calcd for $\mathrm{C}_{20} \mathrm{H}_{24} \mathrm{NO}_{4} \mathrm{Si}[\mathrm{M}+\mathrm{H}]^{+}: 370.1469$, Found: 370.1446 .

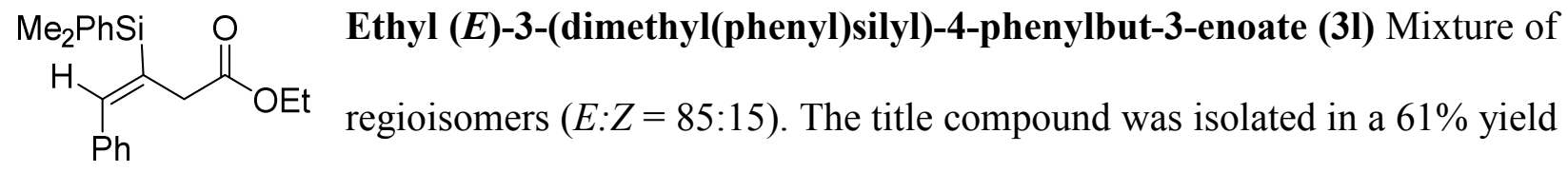

$(55 \mathrm{mg})$ as a clear oil. ${ }^{1} \mathrm{H}$ NMR is consistent with the literature. ${ }^{2}$ HRMS (ESI+): Calcd for $\mathrm{C}_{20} \mathrm{H}_{25} \mathrm{O}_{2} \mathrm{Si}[\mathrm{M}+\mathrm{H}]^{+}: 325.1618$, Found: 325.1628 .

\section{$\mathrm{Me}_{2} \mathrm{PhSi} \quad \mathrm{O} \quad$ Isopropyl (E)-3-(dimethyl(phenyl)silyl)-4-phenylbut-3-enoate (3m) \\ Mixture of regioisomers $(E: Z=85: 15)$. The title compound was isolated in} $73 \%$ yield $(64 \mathrm{mg})$ as a clear oil. ${ }^{1} \mathrm{H}$ NMR $\left(400 \mathrm{MHz}, \mathrm{CDCl}_{3}\right) \delta 7.61-7.55(\mathrm{~m}, 2 \mathrm{H}), 7.38-7.35$ (m, 3H), $7.34-7.31(\mathrm{~m}, 3 \mathrm{H}), 7.22-7.29(\mathrm{~m}, 1 \mathrm{H}), 7.10-7.18(\mathrm{~m}, 1 \mathrm{H}), 7.06-7.02(\mathrm{~s}, 1 \mathrm{H}), 4.96-$ $4.87(\mathrm{~m}, 1 \mathrm{H}), 3.28(\mathrm{~s}, 2 \mathrm{H}), 1.16(\mathrm{~d}, J=8 \mathrm{~Hz}, 6 \mathrm{H}), 0.47(\mathrm{~s}, 6 \mathrm{H}) ;{ }^{13} \mathrm{C} \mathrm{NMR}\left(101 \mathrm{MHz}, \mathrm{CDCl}_{3}\right) \delta$ $171.7,147.4,143.5,137.9,137.6,135,134.3,134.1,129.3,128.8,128.7,128.3,127.9,127.8$, 127.4, 68.2, 68.02, 44.5, 36.6, 21.9, -1.0, -2.5; HRMS (ESI+): Calcd for $\mathrm{C}_{21} \mathrm{H}_{27} \mathrm{O}_{2} \mathrm{Si}[\mathrm{M}+\mathrm{H}]^{+}$ 339.1775, Found: 339.1748 .

\footnotetext{
$\begin{array}{ll}\mathrm{Me}_{2} \mathrm{PhSi} & \text { Benzyl (E)-3-(dimethyl(phenyl)silyl)-4-phenylbut-3-enoate (3n) } \\ \mathrm{OCH}_{2} \mathrm{Ph} & \text { Mixture of regioisomers (E:Z: 86: 14). The title compound was isolated }\end{array}$ in a $75 \%$ yield $(76 \mathrm{mg})$ as a clear oil. ${ }^{1} \mathrm{H}$ NMR $\left(400 \mathrm{MHz}, \mathrm{CDCl}_{3}\right) \delta 7.56-7.54(\mathrm{~m}, 2 \mathrm{H}), 7.45-$ $7.10(\mathrm{~m}, 13 \mathrm{H}), 7.07$ (s, 1H), 4.96 (s, 2H), $3.35-3.35$ (d, 2H, $J=1.2 \mathrm{~Hz}), 0.44$ (s, 6H); ${ }^{13} \mathrm{C}$ NMR $\left(101 \mathrm{MHz}, \mathrm{CDCl}_{3}\right) \delta 171.8,147.5,143.4,137.6,137.3,135.8,134.6,134.1,133.9,129.1,128.8$
} 
$128.6,128.5,128.4,128.3,128.2,128.1,127.7,127.6,127.2,66.4,66.3,44,36.1,-1.3,-2.9$; HRMS (ESI+): Calcd for $\mathrm{C}_{25} \mathrm{H}_{30} \mathrm{NO}_{2} \mathrm{Si}\left[\mathrm{M}+\mathrm{NH}_{4}\right]^{+}$404.204, Found: 404.2048 . 


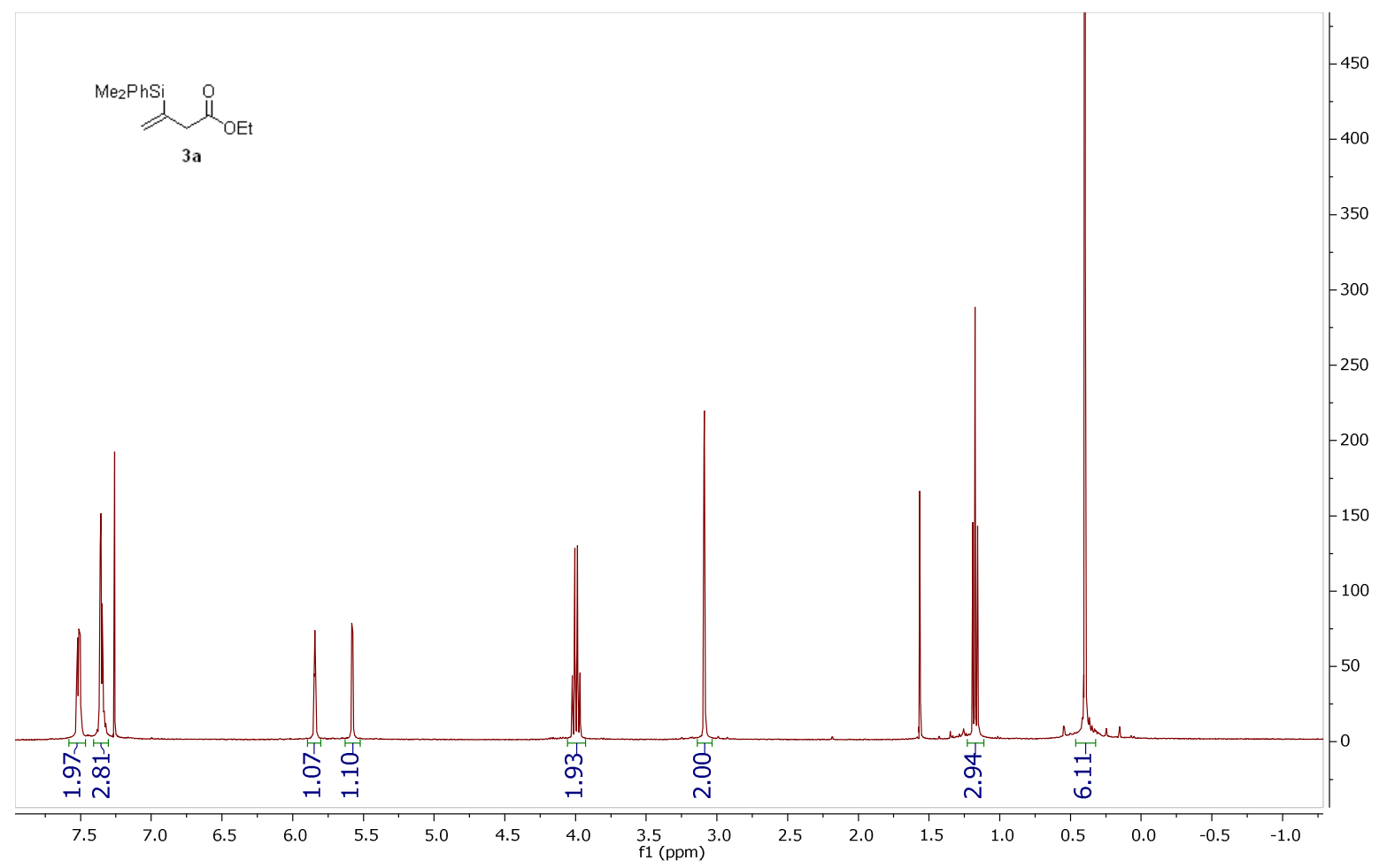




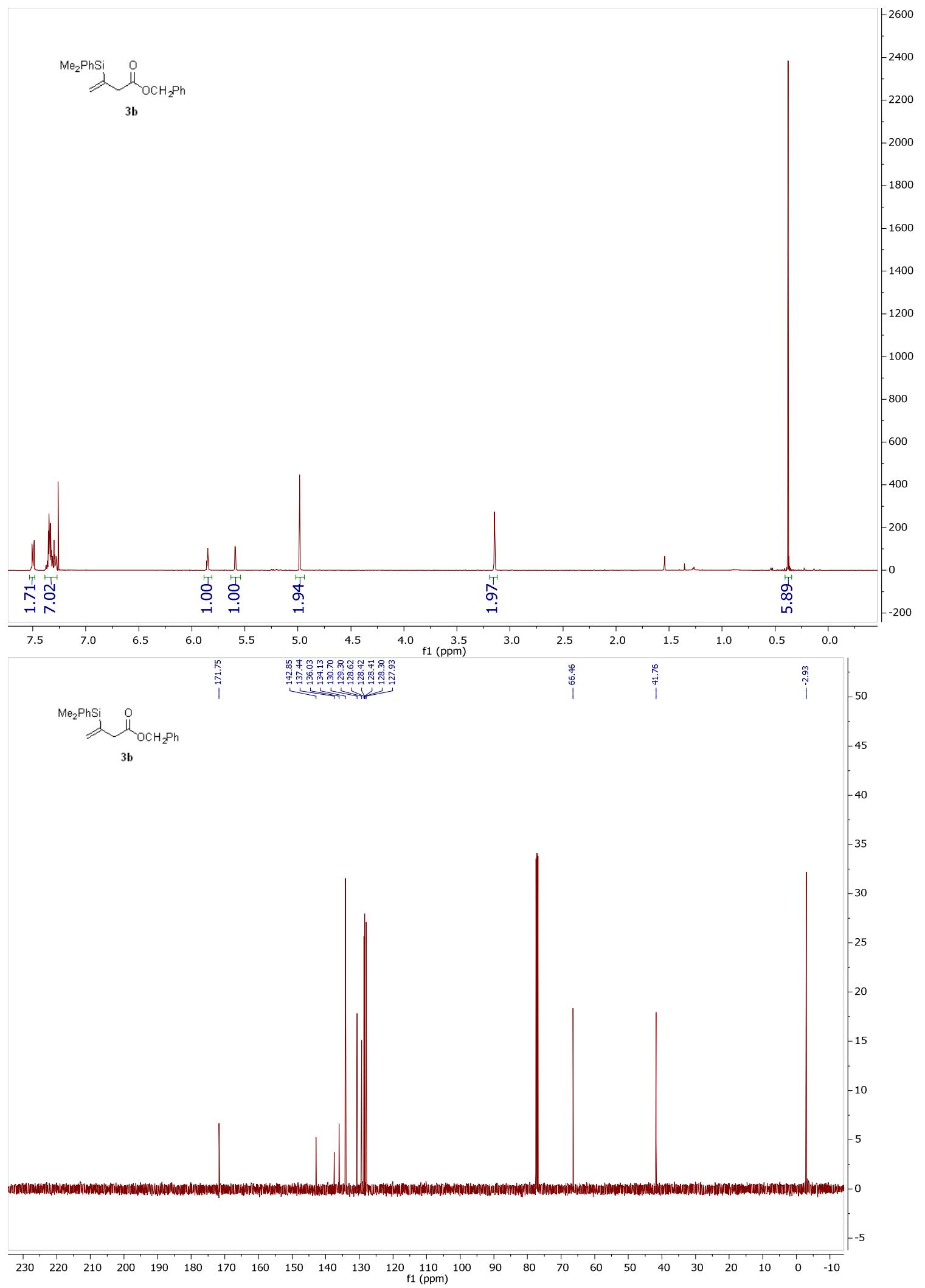



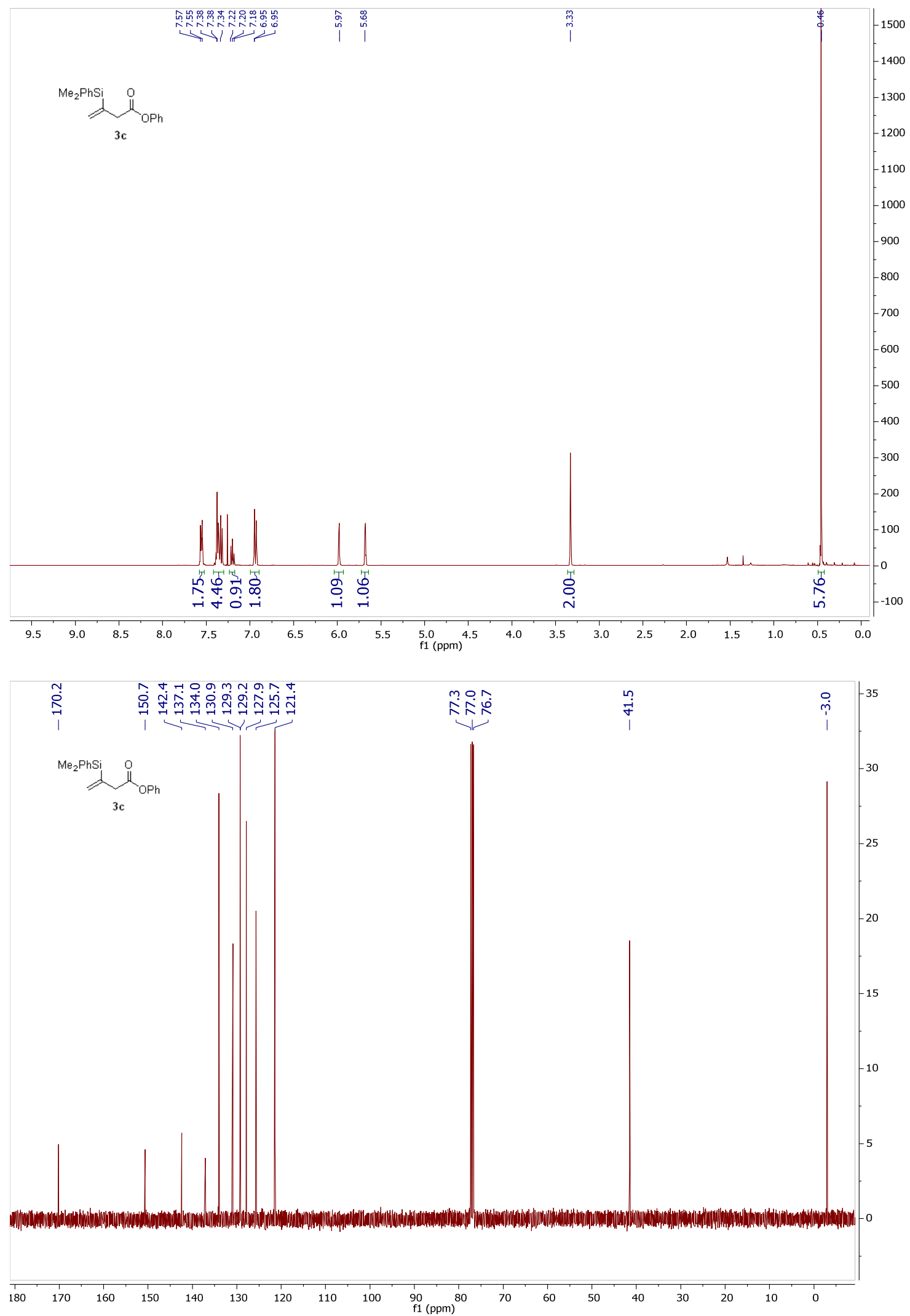


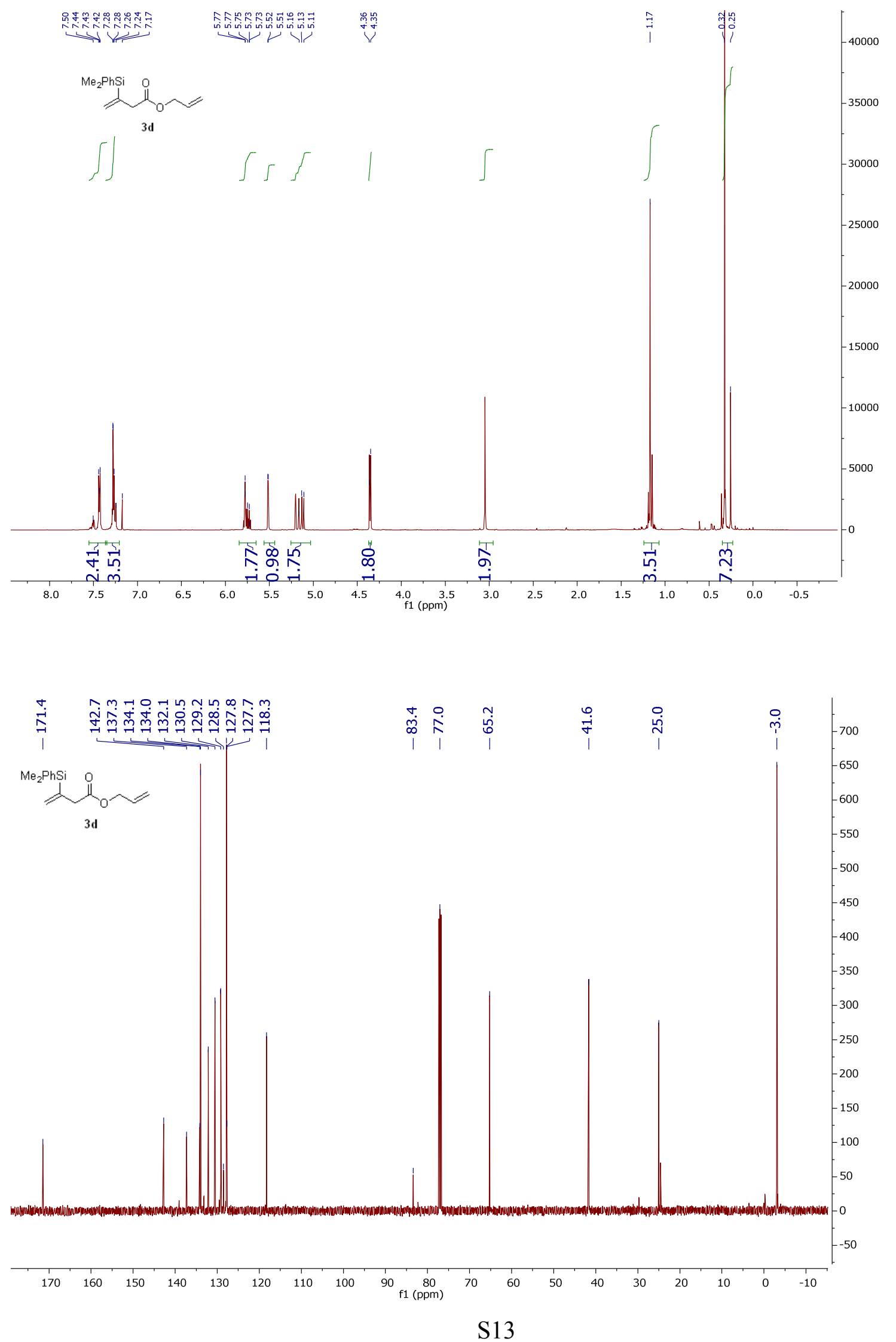




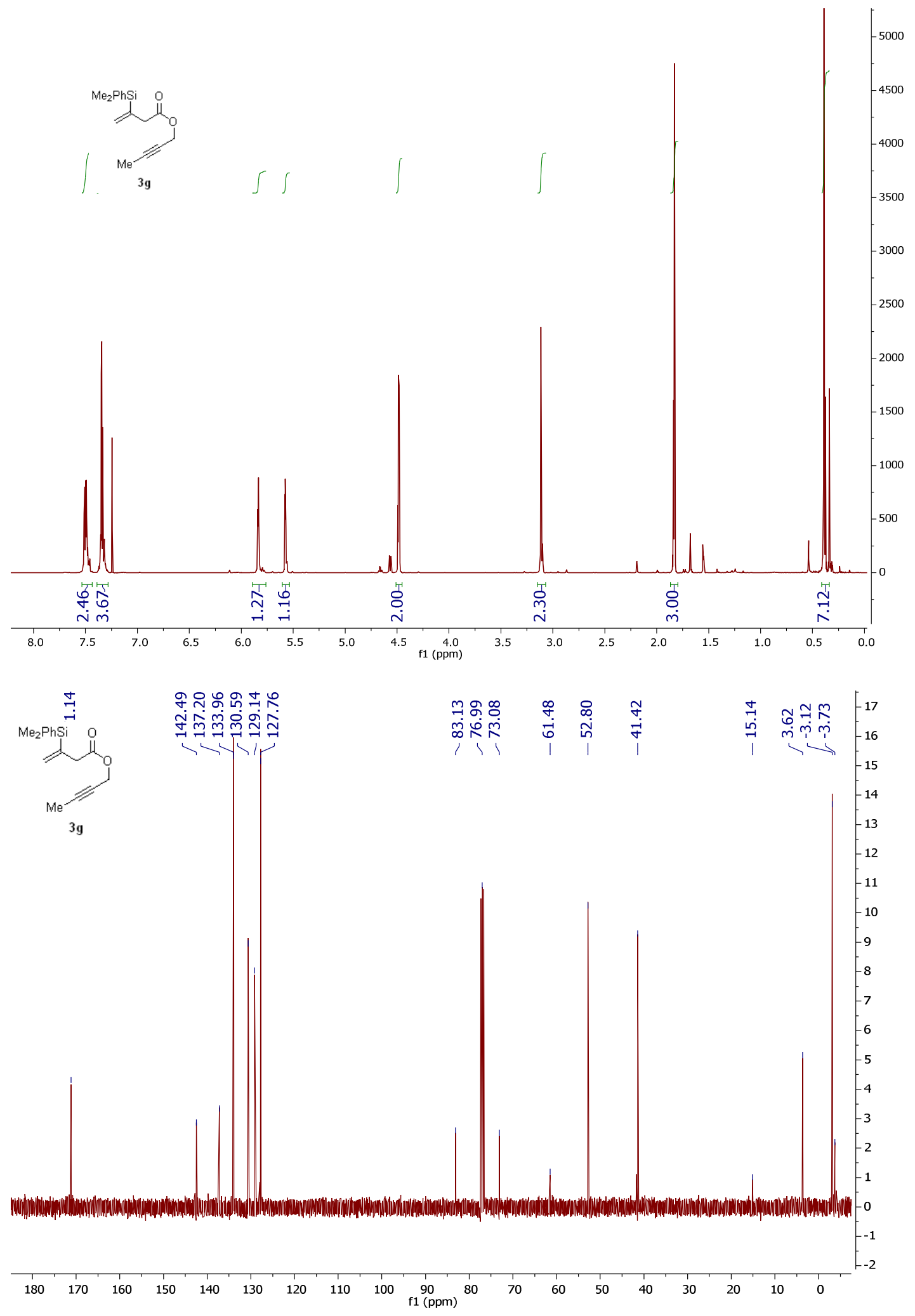




\section{Compound 3h}
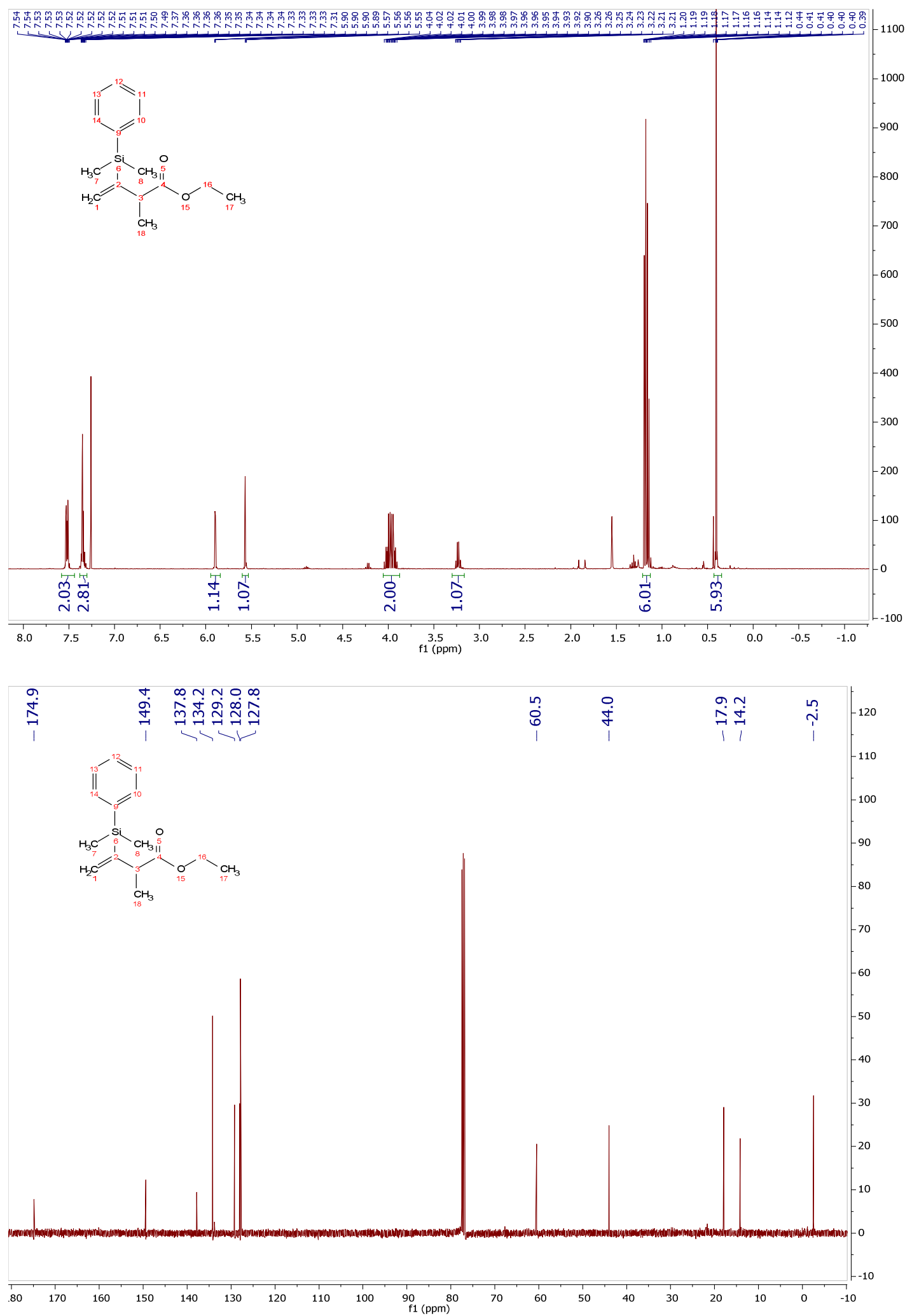

S15 

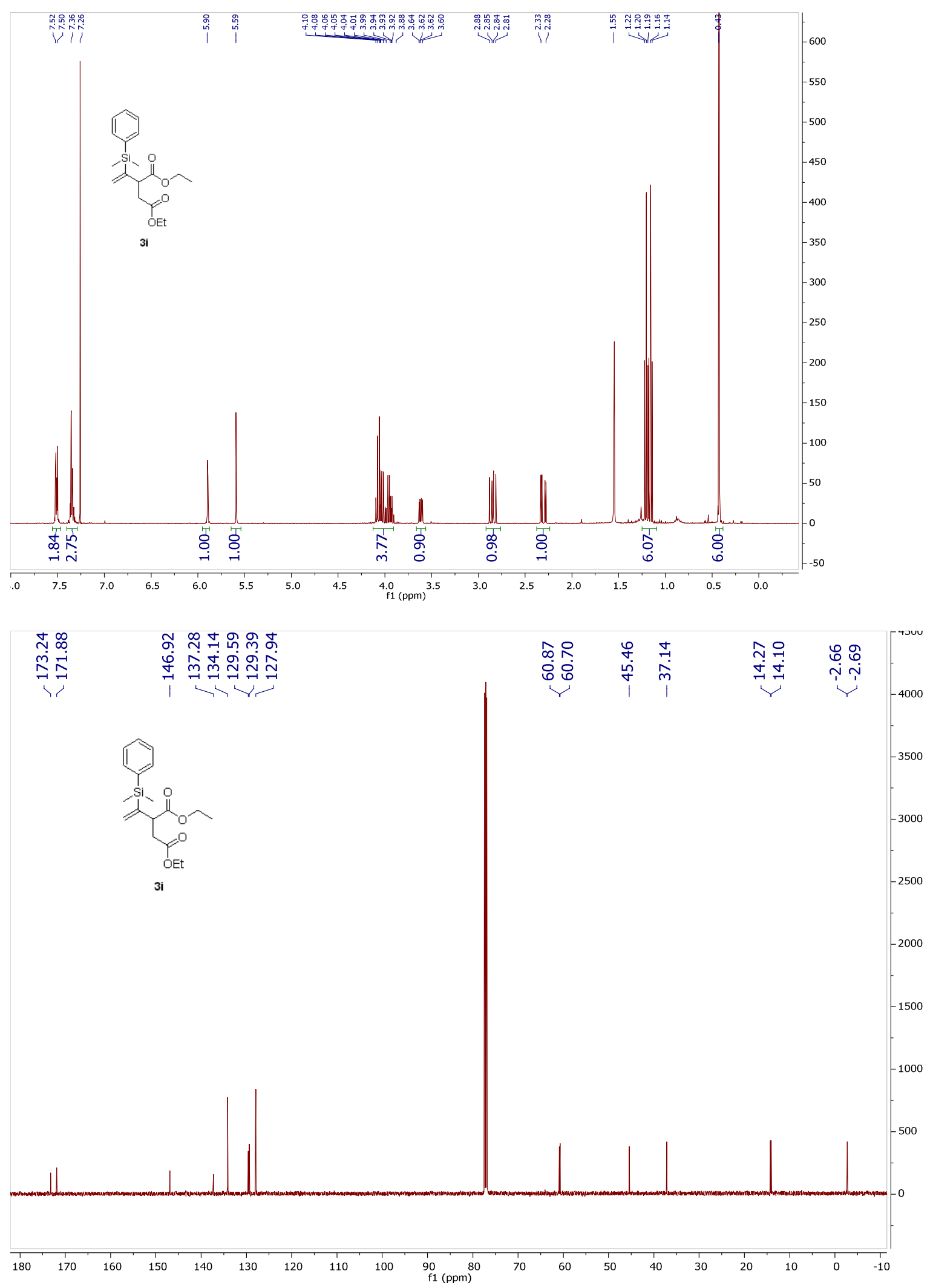


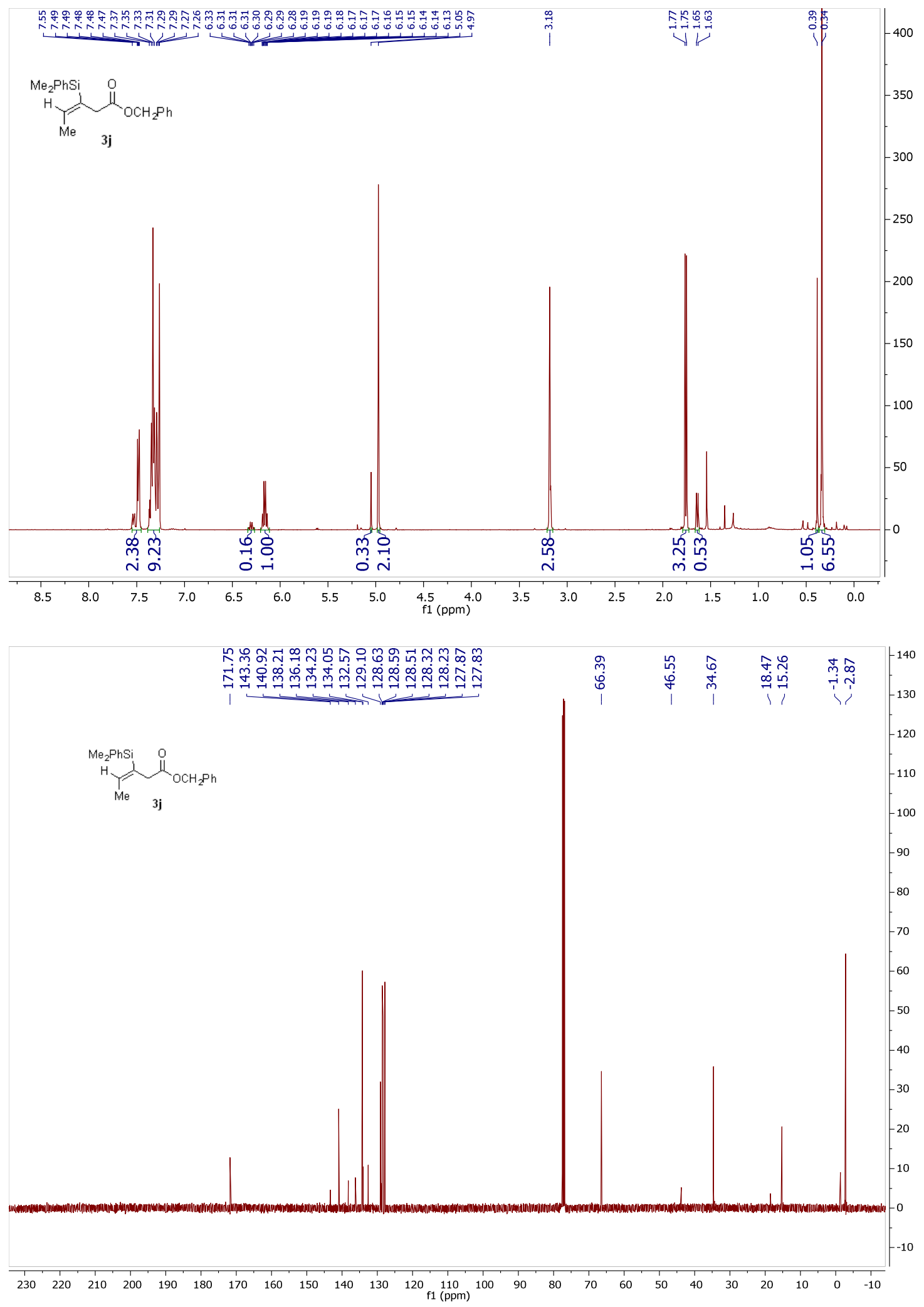



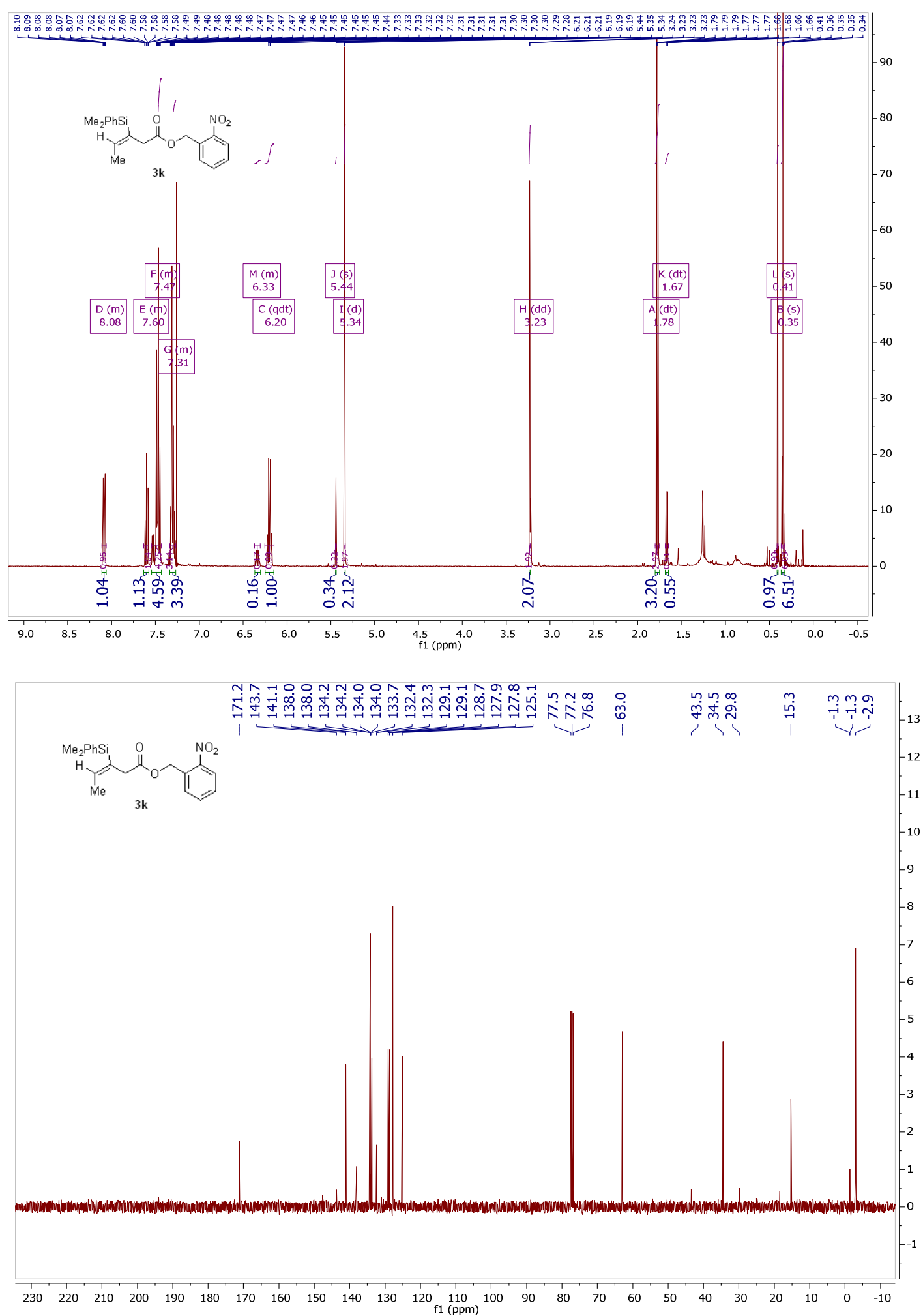


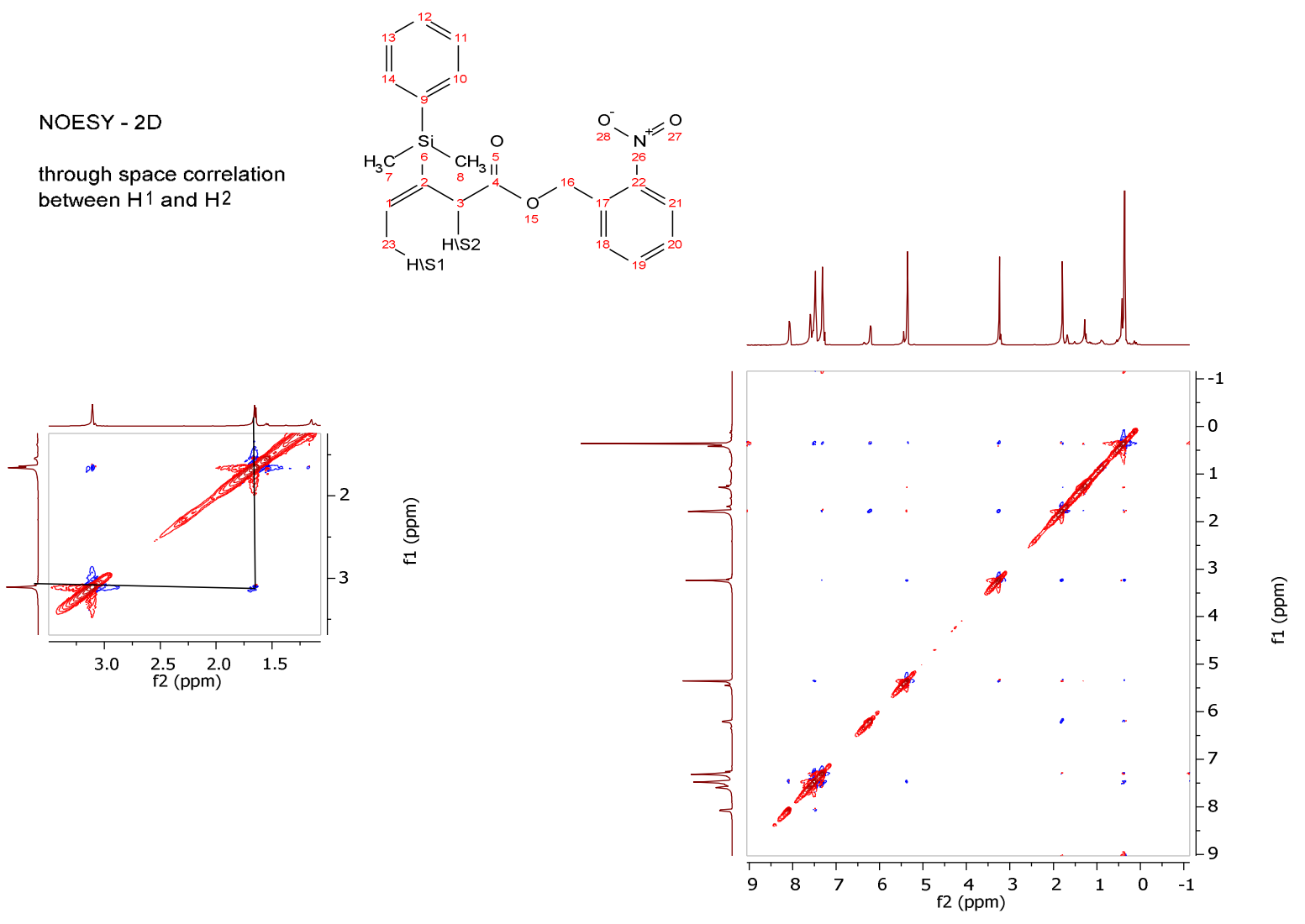




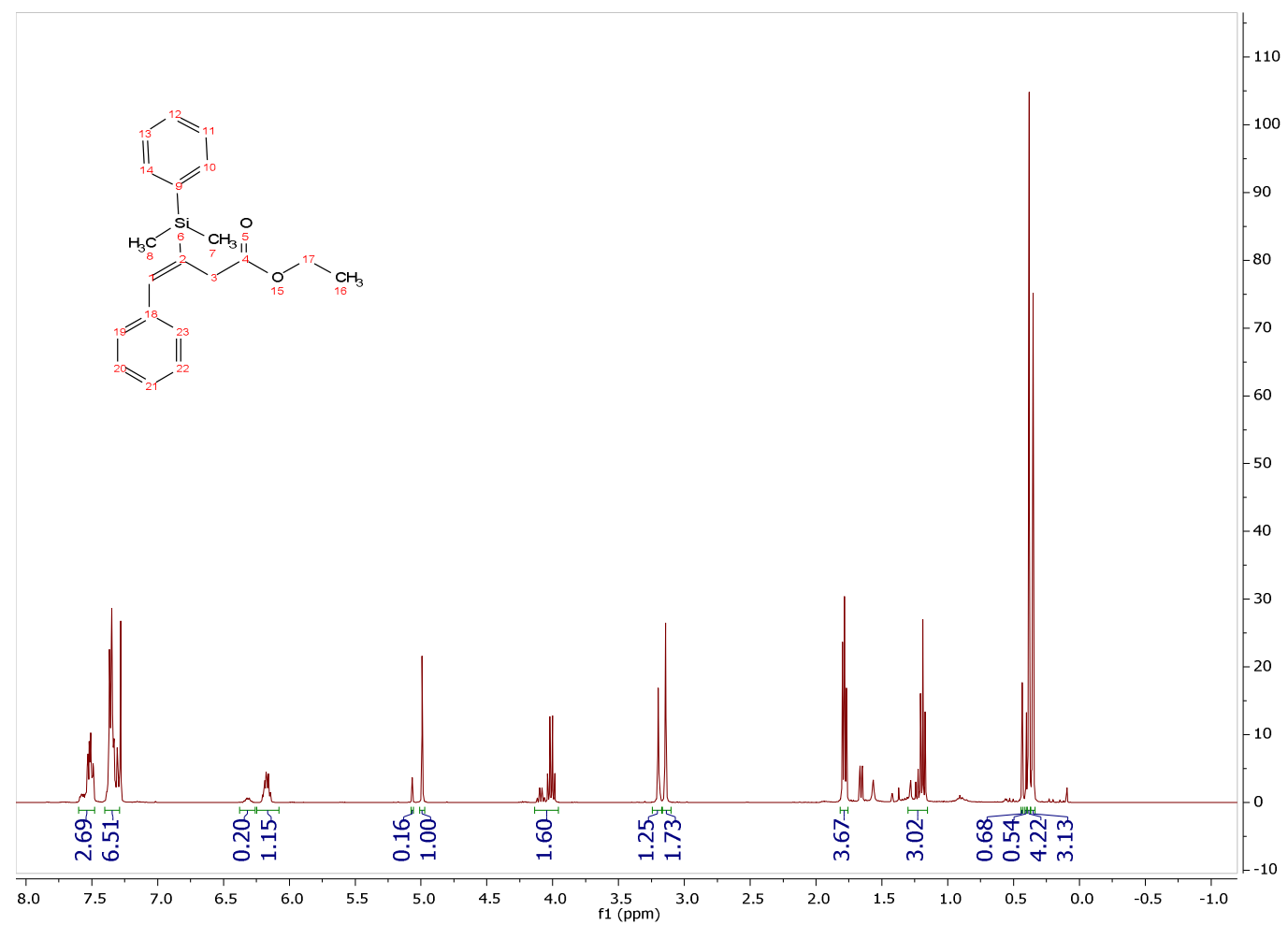




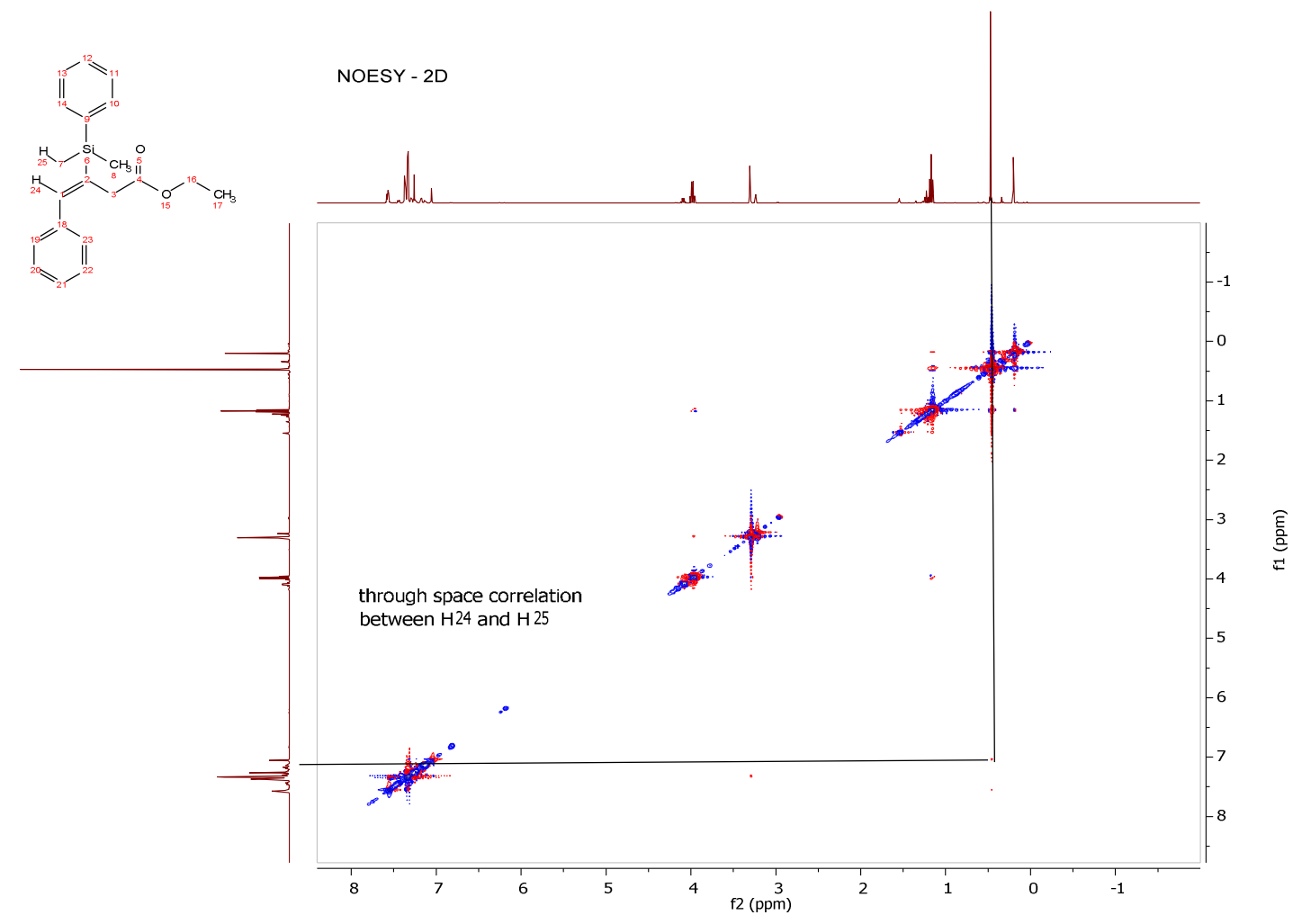



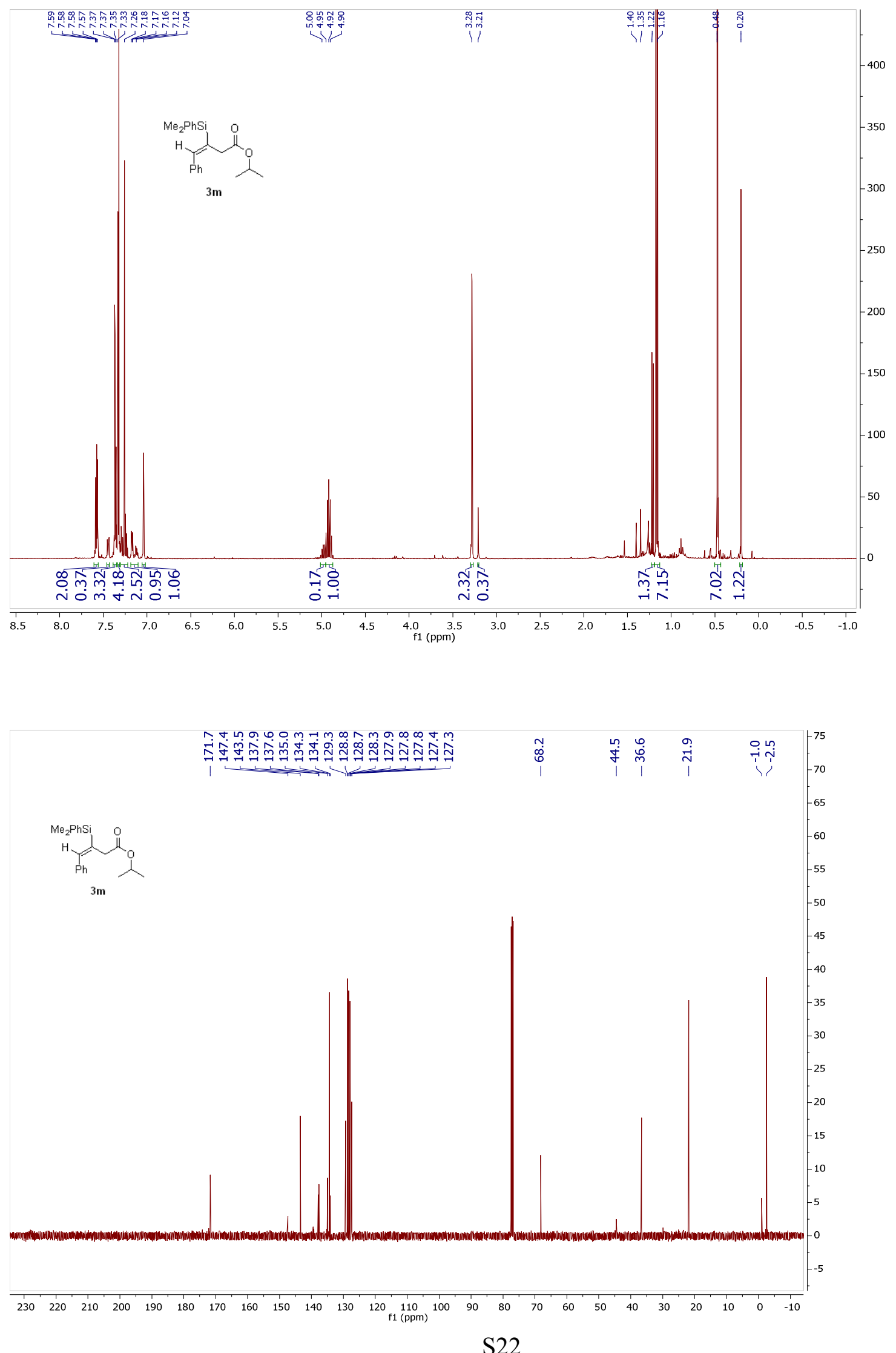


\section{Compound 3m}

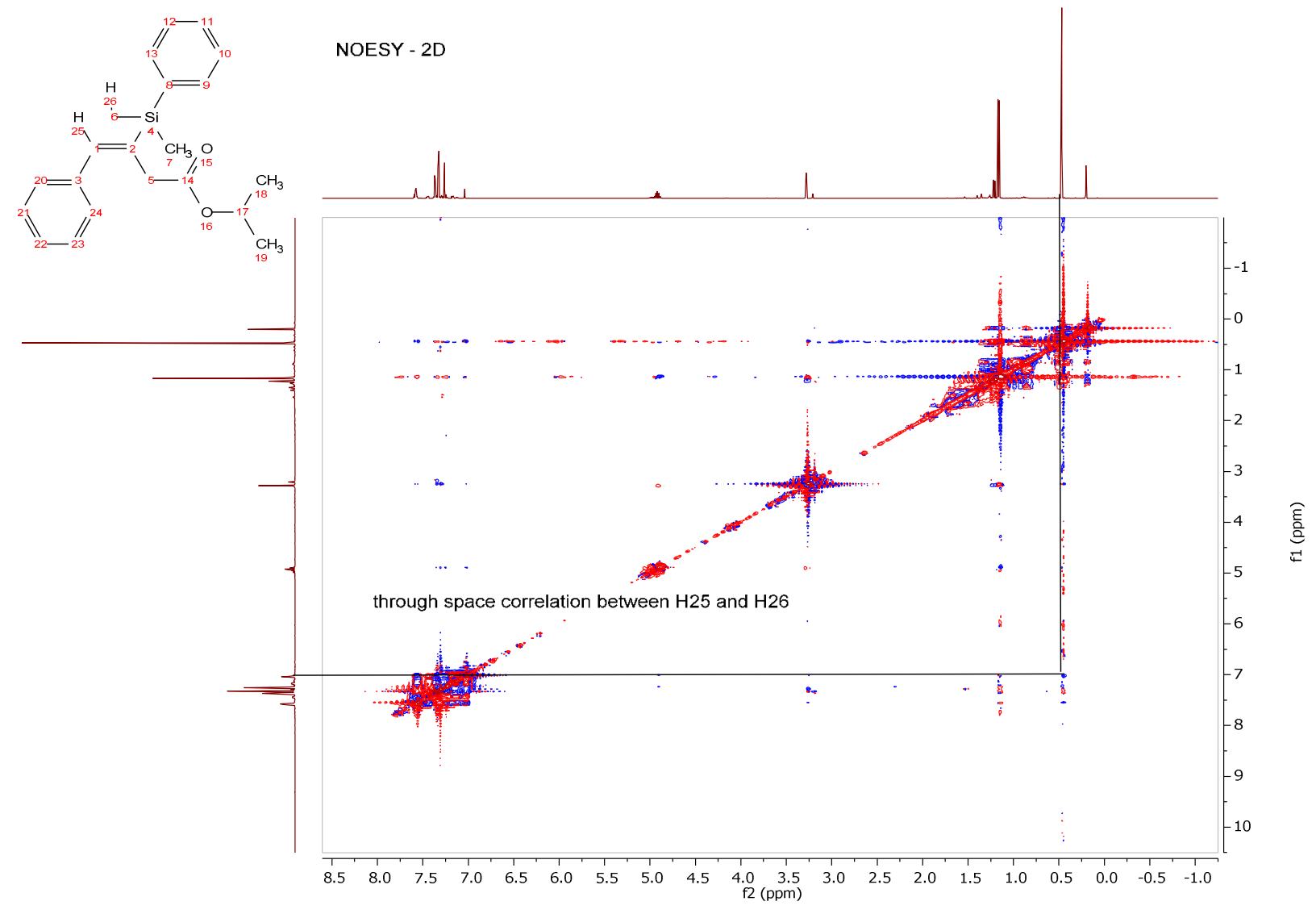



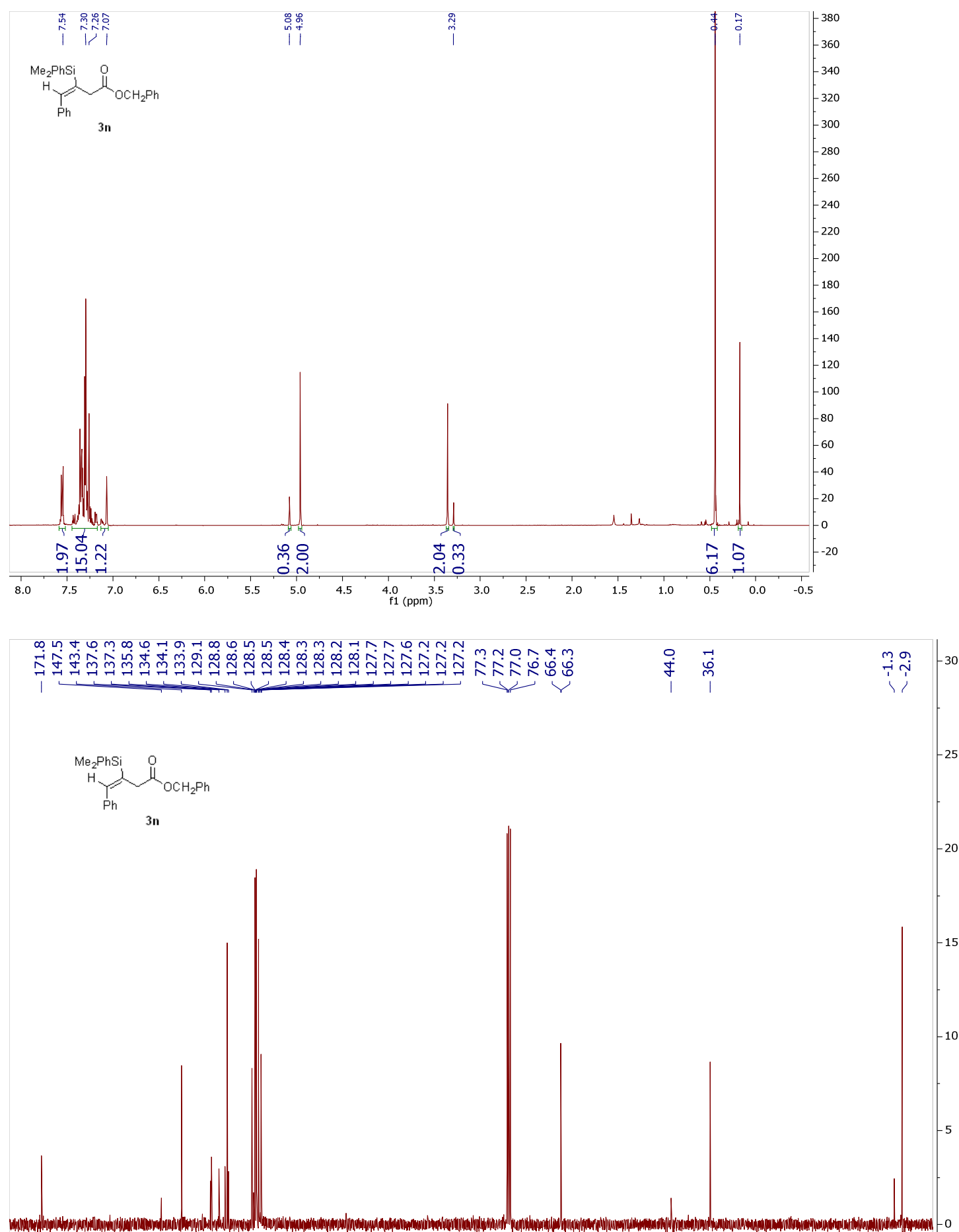


\section{Compound 3n}

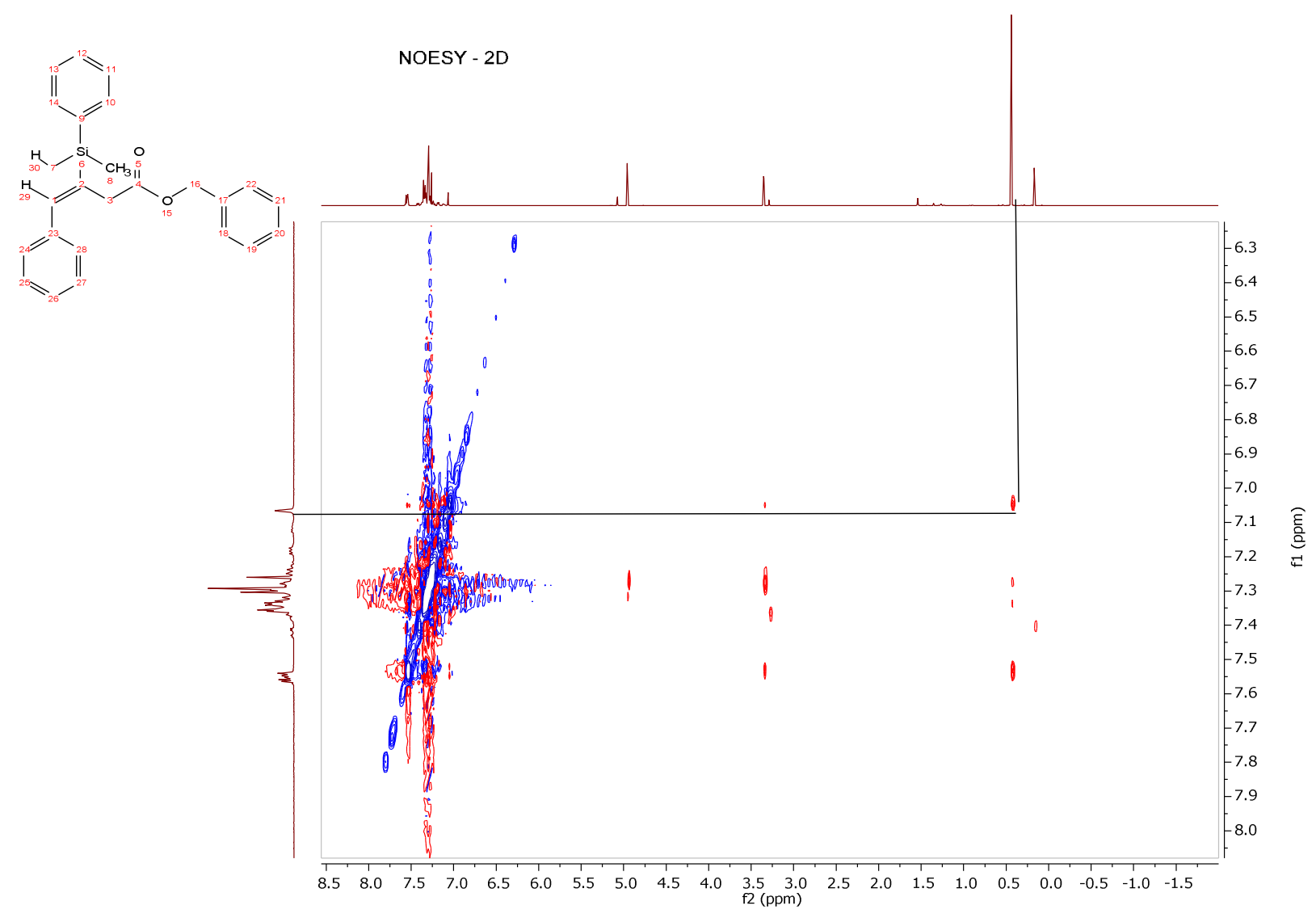

\section{References}

1. (a) Zhu, X. F.; Henry, C. E.; Wang, J.; Dudding, T.; Kwon, O., Phosphine-catalyzed synthesis of 1,3-dioxan-4-ylidenes. Org Lett 2005, 7 (7), 1387-90; (b) Thorpe, S. B.; Guo, X.; Santos, W. L., Regio- and stereoselective copper-catalyzed [small beta]-borylation of allenoates by a preactivated diboron. Chem. Commun. 2011, 47 (1), 424-426.

2. Xu, Y.-H.; Wu, L.-H.; Wang, J.; Loh, T.-P., Synthesis of multi-substituted vinylsilanes via copper(i)-catalyzed hydrosilylation reactions of allenes and propiolate derivatives with silylboronates. Chem. Commun. 2014, 50 (54), 7195-7197. 